\title{
Diagnostic imaging of dementia with Lewy bodies, frontotemporal lobar degeneration, and normal pressure hydrocephalus
}

\author{
Kazunari Ishii ${ }^{1}$ iD
}

Received: 22 August 2019 / Accepted: 10 September 2019 / Published online: 23 September 2019

(C) Japan Radiological Society 2019

\begin{abstract}
Neuroimaging can provide important biomarkers and is very useful for supporting dementia diagnosis. This review summarizes the neuroimaging findings of dementia with Lewy bodies (DLB), frontotemporal lobar degeneration (FTLD), and normal pressure hydrocephalus (NPH). In DLB, medial temporal atrophy is milder than that of Alzheimer's disease. 2-fluoro-2-deoxy-D-glucose-positron emission tomography and brain perfusion single-photon emission computed tomography demonstrate hypometabolism and hypoperfusion in the occipital lobe, in addition to decreased metabolism and perfusion in the parietotemporal, posterior cingulate, precuneus, and frontal association cortices. The cingulate island sign, which shows relatively spared middle-to-posterior cingulate cortex metabolism compared with precuneus hypometabolism, is proposed to detect DLB in 2-fluoro-2-deoxy-D-glucose-positron emission tomography imaging. Reduced uptake in dopamine transporter imaging and reduced myocardial uptake in iodine-123 metaiodobenzylguanidine cardiac scintigraphy are indicative biomarkers for DLB diagnosis. Characteristic findings of FTLD include dominant frontotemporal atrophy, hypometabolism, and hypoperfusion. Most idiopathic NPH cases demonstrate disproportionally enlarged subarachnoid space hydrocephalus findings, including dilated ventricular systems, enlarged Sylvian fissures, tight sulci in the midline, and a high convexity.
\end{abstract}

Keywords Dementia with lewy bodies · Frontotemporal lobar degeneration $\cdot$ Normal pressure hydrocephalus .

Neuroimaging

\section{Introduction}

The most common cause of dementia is Alzheimer disease's $(\mathrm{AD})$, and a review of neuroimaging for $\mathrm{AD}$, especially biomarker imaging [e.g., amyloid positron emission tomography (PET) and tau PET], is described in a previous volume of this journal by Matsuda et al. [1]. Herein, this review describes the characteristic imaging features of dementia with Lewy bodies (DLB), the second most common cause of dementia; frontotemporal lobar degeneration (FTLD), which includes the well-known Pick disease; and normal pressure hydrocephalus (NPH), which occurs in approximately $10 \%$ of ageing dementia cases.

Kazunari Ishii

ishii@med.kindai.ac.jpfig

1 Department of Radiology, Kindai University Faculty of Medicine, 377-2 Ohnohigashi, Osakasayama, Osaka 589-8511, Japan

\section{DLB}

DLB is the second most common cause of neurodegenerative dementia after AD. Its pathological features include the presence of Lewy bodies in cortical, subcortical, and brain stem structures. DLB is a type of Lewy body disease, which includes Parkinson's disease (PD), PD with dementia (PDD) and DLB. PDD has the same pathology as DLB, but clinical Parkinsonism appears $>1$ year earlier than cognitive dysfunction, in contrast with DLB.

DLB diagnostic criteria were revised in 2017 [2]. The core clinical features of DLB include fluctuating cognitive function, recurrent visual hallucinations, Parkinsonian symptoms, and rapid eye movement (REM) sleep behavior disorder. Indicative biomarkers include reduced dopamine transporter uptake in the basal ganglia demonstrated by single-photon emission computed tomography (SPECT) or PET, low cardiac uptake of ${ }^{123}$ I-meta-iodobenzylguanidine ( ${ }^{123}$ I-MIBG) in myocardial scintigraphy, and polysomnographic confirmation of REM sleep without atonia. Supportive biomarkers include relative preservation of medial 
temporal lobe structures on computed tomography/magnetic resonance imaging (MRI), generalized low uptake

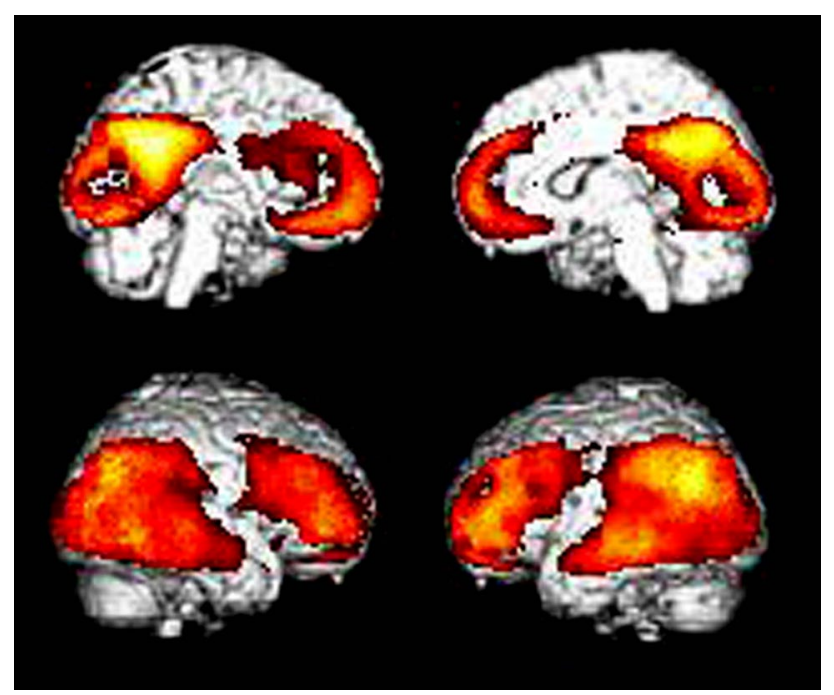

Fig. 1 Reduced glucose metabolic regions of dementia with Lewy bodies (DLB). The regions with significantly decreased glucose metabolism compared with age-matched healthy subjects by statistical parametric maps are shown, and include the parietotemporal and frontal association area, the posterior cingulate gyri, and the occipital cortices on SPECT/PET perfusion/metabolism scan with reduced occipital activity (Fig. 1) and the cingulate island sign (CIS) on 2-fluoro-2-deoxy-D-glucose (FDG)-PET imaging, and prominent posterior slow-wave activity on electroencephalogram with periodic fluctuations in the pre-alpha/ theta range. In the revised criteria of DLB, the imaging feature of low cardiac uptake of ${ }^{123}$ I-MIBG in myocardial scintigraphy was upgraded from a supportive biomarker to an indicative biomarker.

\section{Medial temporal atrophy}

In DLB, medial temporal atrophy was reported to be milder than that in AD [3], although the medial temporal volume of DLB patients is significantly smaller than that of normal aged people [4]. Further, whether the DLB dorsal midbrain volume is significantly smaller than that in AD [5] remains controversial [4]. It is difficult to differentiate individual patients with DLB from those with AD using MRI assessment of medial temporal atrophy (Fig. 2a) or dorsal midbrain atrophy, because $>50 \%$ of patients with DLB have AD pathology.
Fig. 2 A representative case of DLB. a T1-weighted magnetic resonance imaging (MRI) of a patient with DLB. Mild hippocampal and diffuse cortical atrophy are demonstrated. b ${ }^{123}$ I-Iodoamphetamine (IMP) brain perfusion image showing decreased perfusion in the bilateral occipital, parietotemporal, frontal, posterior cingulate, and precuneus cortices a

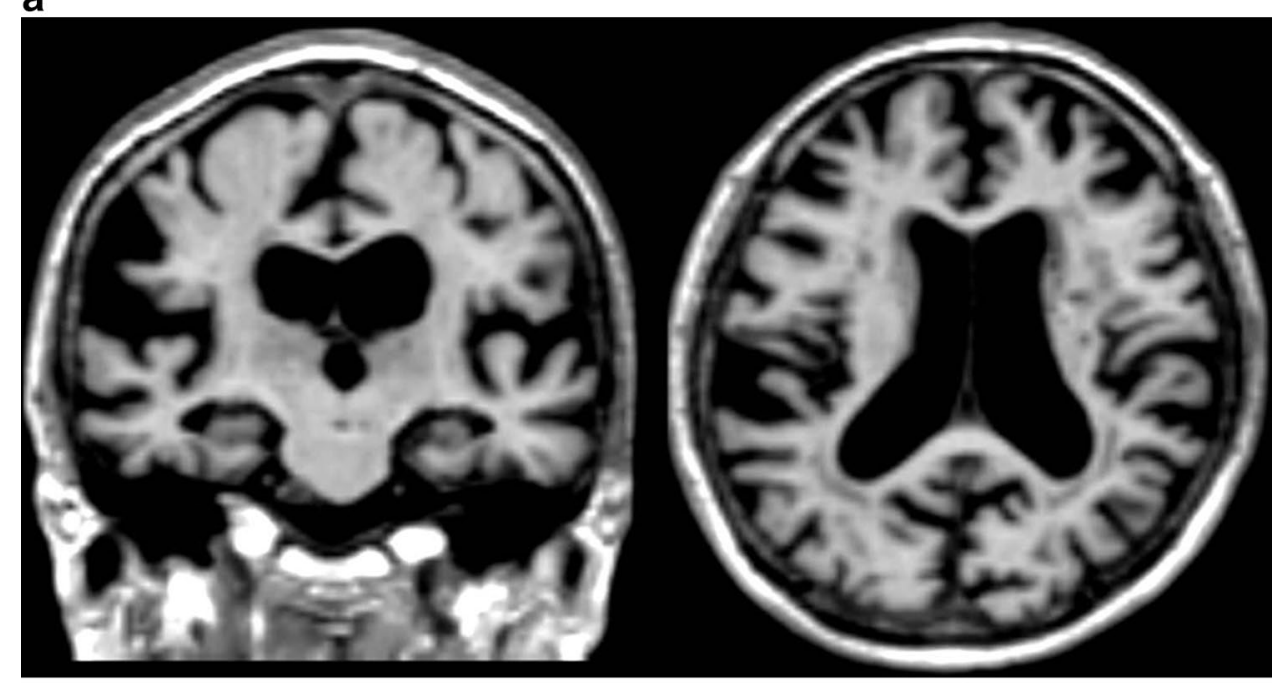

b

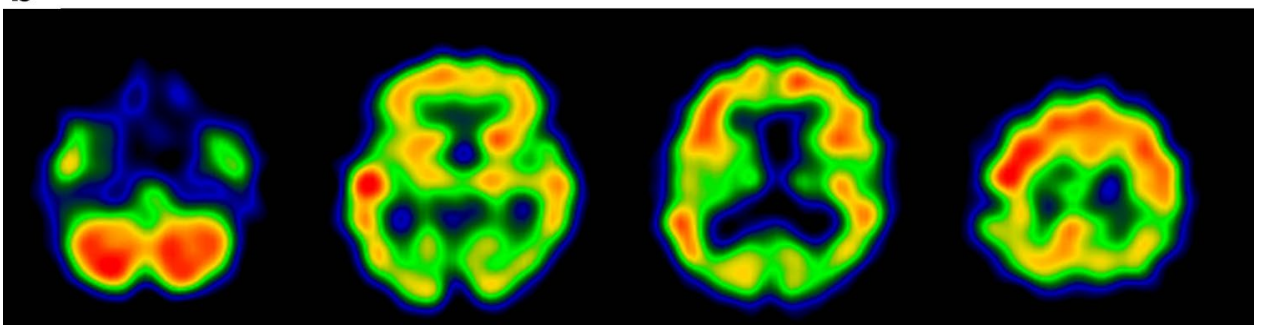




\section{Cerebral perfusion and glucose metabolism}

It is well established that occipital blood flow and glucose metabolism are decreased in DLB (Figs. 2, 3, 4), but preserved in AD [6-10]. FDG-PET is superior to brain perfusion SPECT for differential diagnosis of degenerative dementia by detecting hypofunction in the occipital lobes [11-13]. In DLB, cerebral hypometabolism and hypoperfusion are more severe than those in $\mathrm{AD}$, despite only mild cognitive impairment. Of note, voxel-based analysis may underestimate the degree of regional hypometabolism and hypoperfusion in DLB brain. Since in DLB brain whole

\section{a}

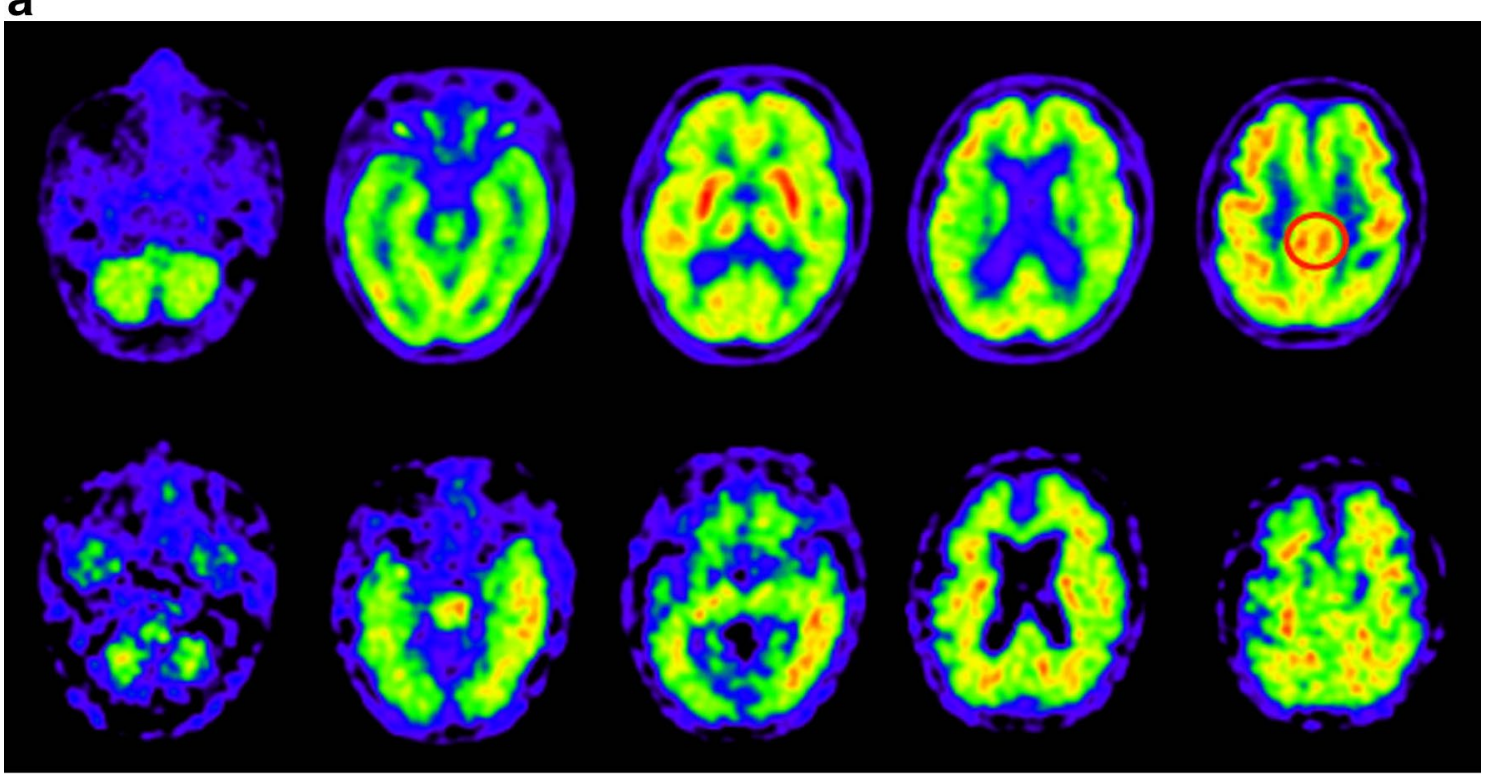

b

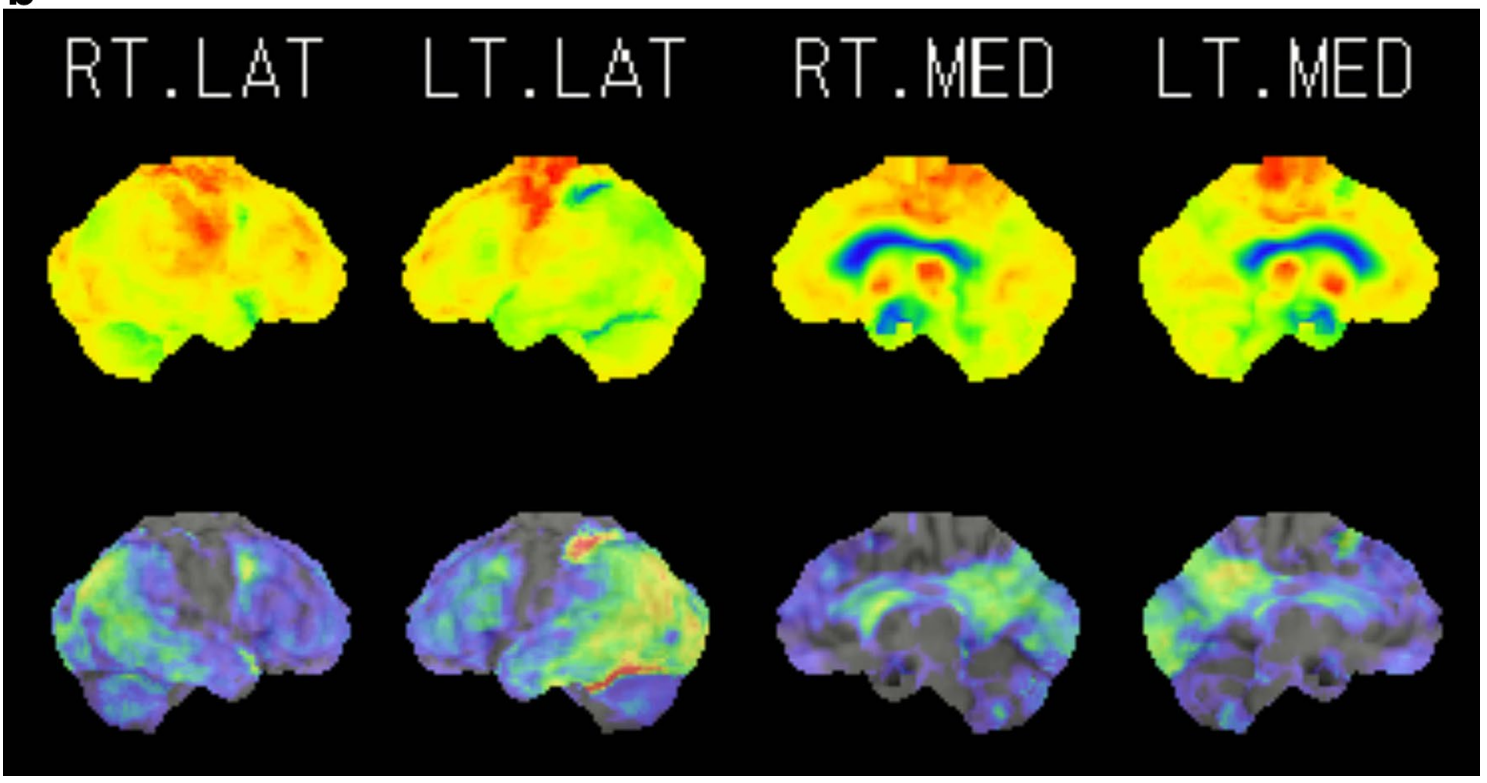

Fig. 3 Representative ${ }^{18}$ F-fluorodeoxy glucose (FDG)-positron emission tomography (PET) images of DLB. a FDG-PET (upper row) and ${ }^{11} \mathrm{C}$-Pittsburgh Compound B (PiB)-PET (lower row) images of a patient with DLB. Diffuse reductions in cortical glucose metabolism, except for the striata and sensorimotor cortices, are demonstrated. The cingulate island sign (red circle) is shown as a relatively preserved middle cingulate metabolism (upper row). ${ }^{11} \mathrm{C}$-PiB PET shows some accumulation in the left temporal, bilateral frontal, parietal, and precuneus cortices, indicating positive amyloid deposition (lower row). b Three dimensional-stereotactic surface projection (3D-SSP) image produced from the ${ }^{18} \mathrm{~F}$-FDG-PET image of this DLB patient. The surface map (upper row) shows preserved metabolism in the primary sensorimotor cortices, while the $z$ score map (lower row) shows significantly decreased metabolism in the bilateral frontal, parietotemporal, posterior cingulate, precuneus, and occipital cortices 
Fig. 4 A representative case of DLB with negative amyloid pathology. a T1-weighted MRI of a patient with DLB. Coronal and axial images demonstrate mild hippocampal atrophy and mild diffuse cortical atrophy. $\mathbf{b}$ ${ }^{18}$ F-FDG-PET (upper row) and ${ }^{11} \mathrm{C}-\mathrm{PiB}$ (lower row) images of a patient with DLB. Diffuse reductions in cortical glucose metabolism, except for the striata and sensorimotor cortices, are demonstrated. The cingulate island sign (red ellipse) is shown as a relatively preserved metabolism in the middleto-posterior cingulate (upper row). ${ }^{11} \mathrm{C}$-PiB PET shows only non-specific accumulation in the white matter and the pons, indicating negative amyloid deposition (lower row). c 3D-SSP image produced from the ${ }^{18}$ F-FDG-PET image of this DLB patient. The surface map (upper row) shows preserved glucose metabolism in the primary sensorimotor cortices, the $z$ score map normalized to the whole brain (middle row) shows significantly decreased metabolism in the bilateral frontal, parietotemporal, posterior cingulate, precuneus, and occipital cortices, while the $z$ score map normalized to the cerebellum (lower row) shows a much larger area of decreased metabolism compared with the $z$ score map normalized to the whole brain a

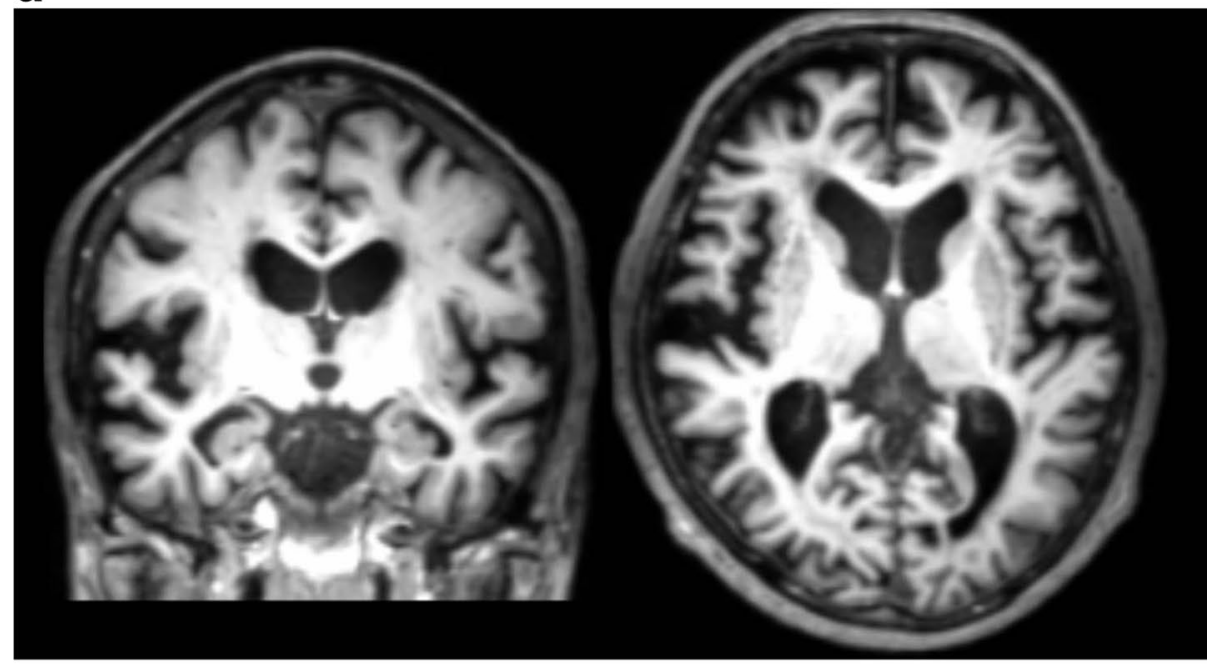

b

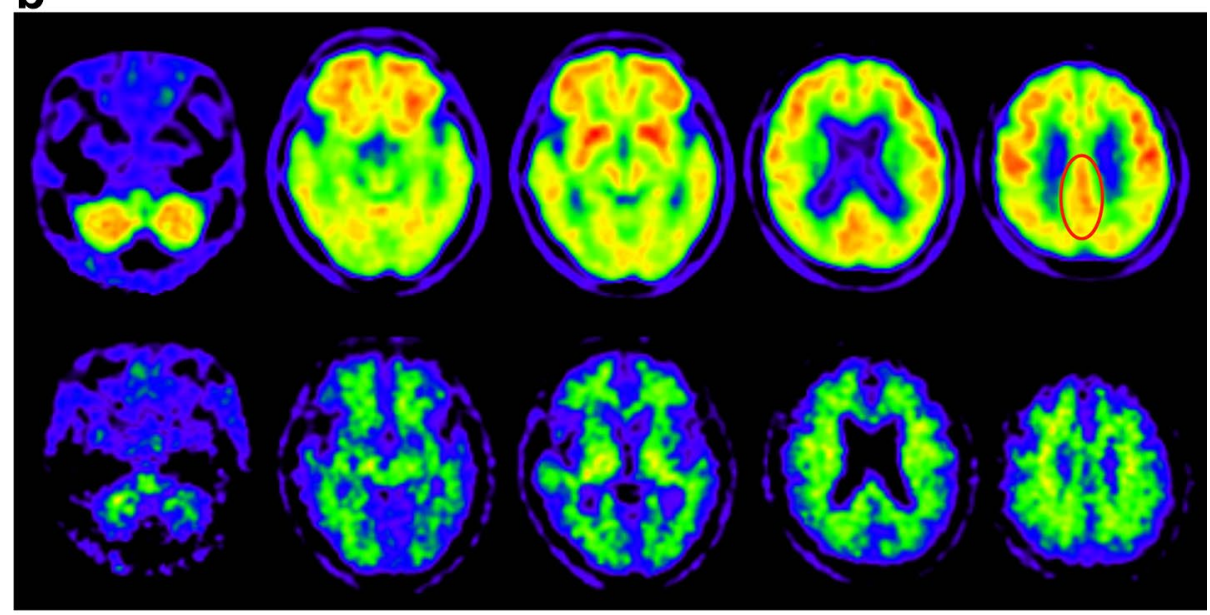

C

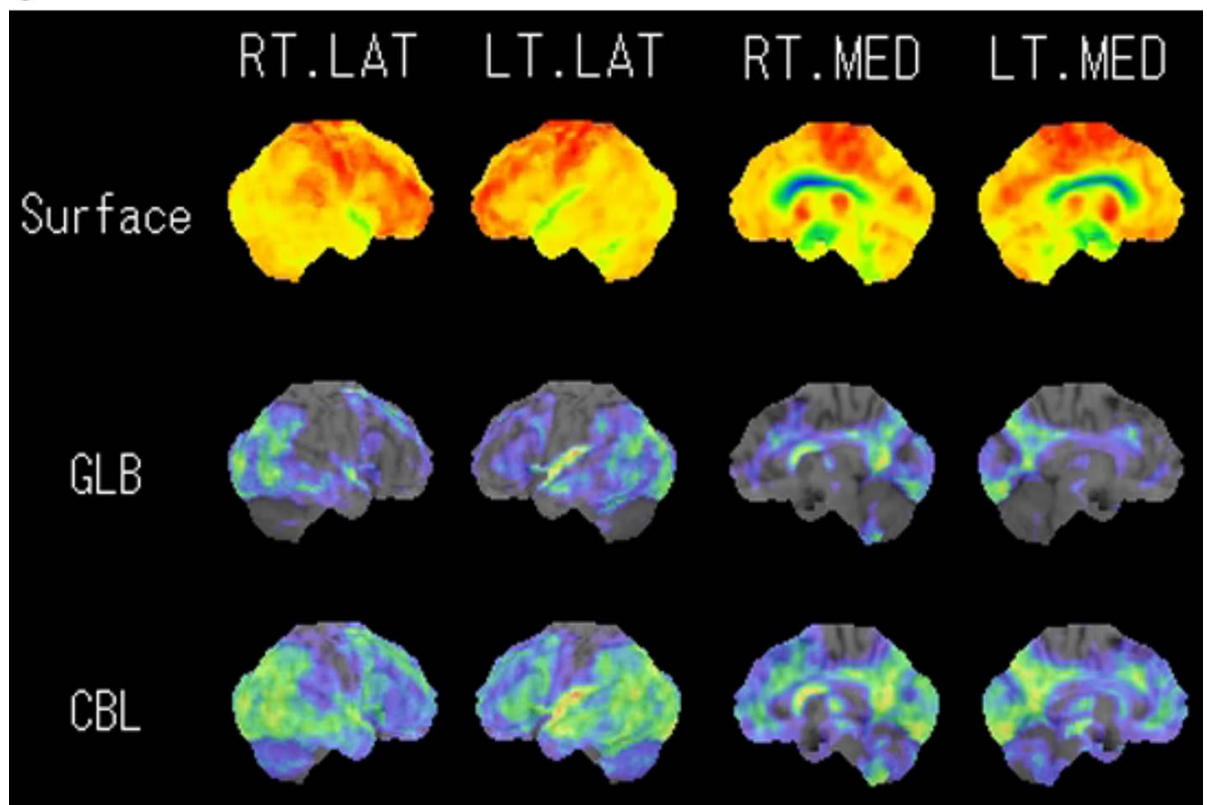


cerebral metabolism and perfusion are often decreased globally, when the regional relative metabolic or perfusion value is calculated as regional activity counts divided by global activity counts, the relative value becomes larger. Therefore, in calculating the regional relative value, cerebellar activity counts as a reference region is preferable to global activity counts as a reference (Fig. 4c). In clinical practice, computer-assisted diagnosis (CAD) systems are available [14], but it is important that we should use these systems for diagnosis assistance with visual inspection, never make diagnoses using only these CAD systems.

\section{CIS}

CIS (Fig. 3a) was reported to be characterized by a spared mid-posterior cingulate glucose metabolism compared with the precuneus metabolism [15]. However, this phenomenon of a relatively higher glucose metabolism in the middle cingulate gyrus in DLB subjects compared with AD subjects had been already reported [6], although at that time decreased occipital metabolism in DLB brain was emphasized. CIS on SPECT images has been also reported [16]. CIS was recently noted as a good marker for DLB, although because CIS is influenced by concomitant AD pathology, it is not always detected, or it appears/disappears with individual patient prognosis, in DLB [17].

\section{Dopamine transporter imaging and ${ }^{123}$ I-meta iodobenzylguanidine cardiac scintigraphy}

Dopamine transporter imaging with [ $\left.{ }^{123} \mathrm{I}\right] \mathrm{N}$ - $\omega$-fluoropropyl$2 \beta$-carboxymethoxy-3 $\beta$-(4-iodophenyl) nortropane (FP-CIT) SPECT imaging is a useful tool for diagnosing PD and DLB [18]. DLB patients have a significantly lower uptake of
FP-CIT in the caudate nucleus and putamen than that for $\mathrm{AD}$ patients and normal subjects (Fig. 5). Although it is difficult to distinguish other Parkinsonian syndromes using FP-CIT SPECT, such as progressive supranuclear palsy, corticobasal degeneration, or multiple system atrophy, when combined with MRI it may provide good performance for differential diagnosis [19]. DLB patients show no correlation of cognitive function with FP-CIT uptake in any striatal regions, while Parkinsonism is associated with reduced striatal FPCIT uptake [20]. FP-CIT SPECT is useful for diagnosing DLB, but it can be initially rated as normal, then become abnormal over time, in patients with probable DLB, therefore, repeating FP-CIT SPECT may be performed [21].

MIBG myocardial scintigraphy is also an accurate examination for differential diagnosis between DLB and other dementias. The heart/mediastinum ratio of MIBG uptake in DLB patients is significantly lower than that in AD patients (Fig. 6) and control subjects [22]. At the stage of possible DLB, MIBG myocardial scintigraphy can predict the future conversion to probable DLB [23]. Both FP-CIT SPECT and MIBG myocardial scintigraphy have the same diagnostic accuracy in differentiating DLB from AD [24, 25], and the combination of both examinations enables more accurate differentiation between DLB and AD compared with either technique alone [24]. Nevertheless, as combining these modalities is expensive, stand-alone examination should be performed wherever possible to diagnose DLB.

\section{Amyloid imaging for DLB}

Amyloid deposits are associated with AD-like atrophy in patients with DLB [26]. The size of the amyloid deposits, which may be linked to cognitive impairment, are larger in DLB patients than that in PD and PDD [26-30]. Amyloid
Fig. $5{ }^{123} \mathrm{I}-N$ - $\omega$-Fluoropropyl$2 \beta$-carboxymethoxy-3 $\beta$ - $(4-$ iodophenyl) nortropane (FPCIT) single-photon emission computed tomography (SPECT) images of DLB and Alzheimer's disease. In DLB, uptake of FP-CIT is decreased in the bilateral striata, showing a 'dot sign', while uptake is normal in AD FP-CIT, showing a 'comma sign'
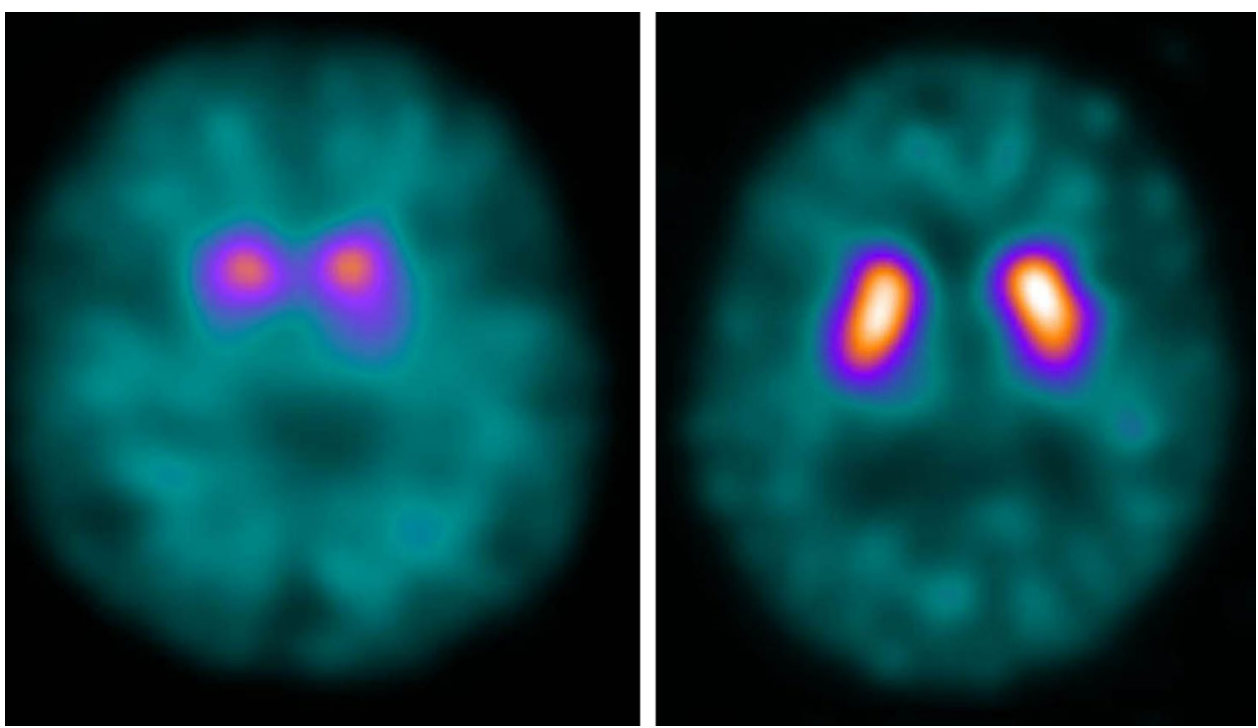
Fig. $6{ }^{123}$ I-Meta-iodobenzylguanidine (MIBG) myocardial scintigraphy of DLB and AD. In DLB, there is nearly no MIBG uptake in the myocardium, while normal MIBG uptake in the myocardium is shown in $\mathrm{AD}$

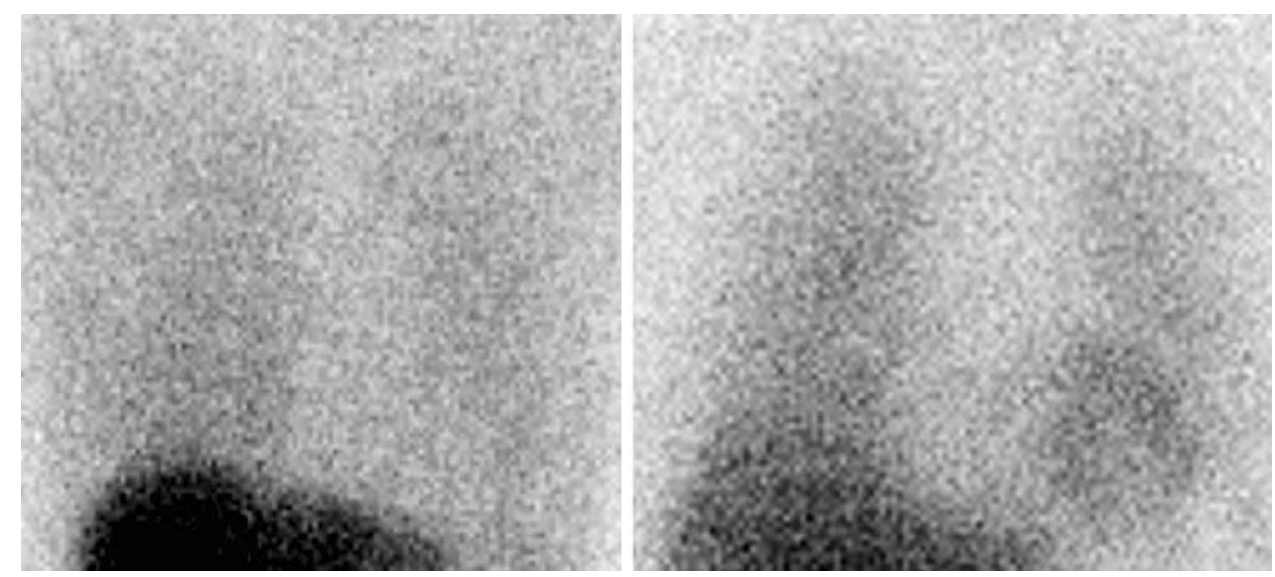

deposition in PDD patients was reported to contribute to memory impairment and faster rates of cognitive decline [31]. However, as shown in Fig. 4b, despite no evidence of amyloid deposits, severe metabolic reductions in some patients with DLB are demonstrated [32].

\section{FTLD}

FTLD is a heterogeneous syndrome consisting of three clinical types of dementia: behavior variant frontotemporal dementia (bvFTD) [33], semantic dementia (SD), and progressive non-fluent aphasia (PNFA) (Fig. 7a) [34]. Pathologically FTLD is classified into various types (Fig. 7b) and overlapping clinical classifications [35-37].

\section{bvFTD}

MRI allows detection of bvFTD-specific brain atrophy, which includes frontal and anterior temporal volume loss, and shows a good diagnostic accuracy for differentiating FTD from normal subjects and other dementias in clinical practice [38-40]. MRI of bvFTD can show severe frontal and anterior temporal atrophy (Figs. 8, 9), while a voxelbased morphometry study [41] showed evidence of metabolic and atrophic changes in the bilateral frontal and temporal lobes in FTD patients, whereas the affected regions of metabolism are more severe and larger than those of atrophy in the frontal and temporal lobe. Further, detecting FTLD using MRI atrophy biomarkers, derived from normalized volumes by automated calculation of the anterior versus posterior index, the asymmetry index, and the temporal pole left index, was reported to provide additional diagnostic assessment and assist in diagnosing FTLD in clinical practice [42].

In FTD, there is a characteristic reduction in frontal and anterior temporal glucose metabolism, although medial temporal, striatal, and thalamic metabolism are also affected [43-45]. Arterial spin labelling (ASL) MRI can provide
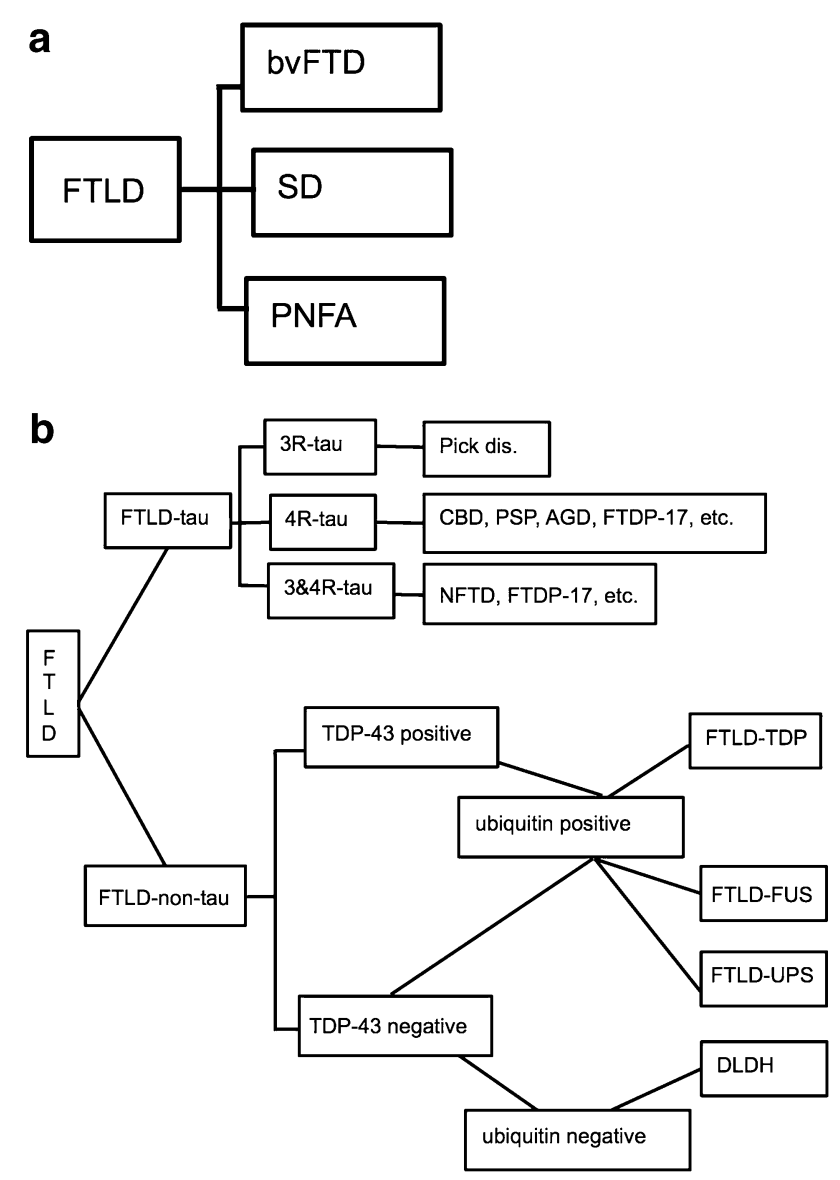

Fig. 7 Classification of frontotemporal lobar degeneration (FTLD). a Clinical classification of FTLD. b Pathological classification of FTLD. $b v F T D$ behavioral variant frontotemporal dementia, $S D$ semantic dementia, $P N F A$ progressive non-fluent aphasia, $3 R$ three repeat, $4 R$ four repeat, $C B D$ corticobasal degeneration, $P S P$ progressive supranuclear palsy, $A G D$ argyrophilic grain disease, TDP TAR DNA binding protein, FTDP frontotemporal degeneration and Parkinsonism, FUS fused in sarcoma, UPS ubiquitin-proteasome system, $D L D H$ dementia lacking distinctive histology 
Fig. 8 Reduced gray matter volumes in FTLD. Gray matter regions showing significant volume decreases compared with age-matched healthy subjects by statistical parametric maps are shown, indicating frontal and anterior temporal gray matter atrophy
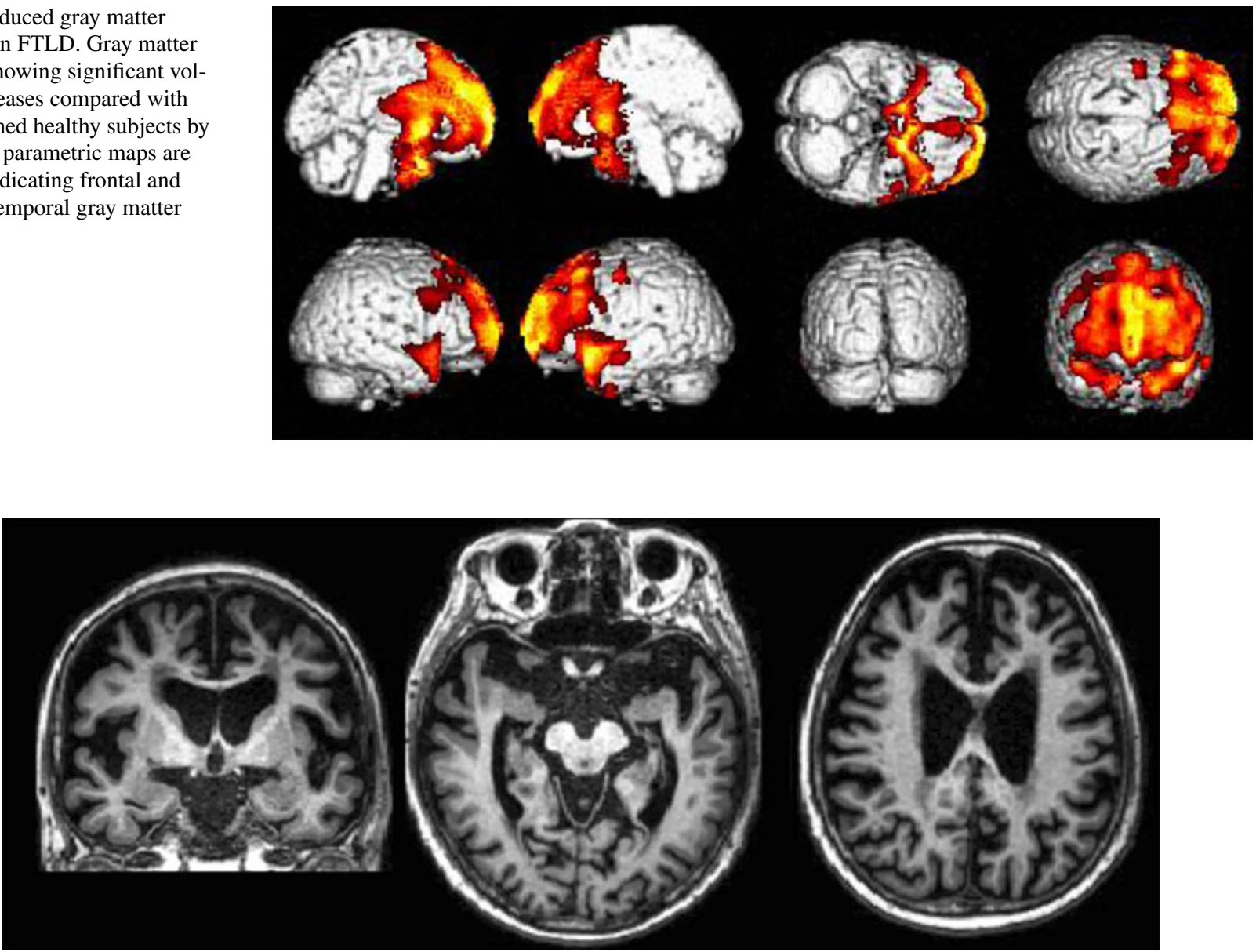

Fig. 9 Representative case of bvFTD. Bilateral frontal and anterior temporal atrophy are shown on T1-weighted MRI

brain perfusion images, and was verified for diagnosing FTD [45]. However, the accuracy for discriminant diagnosis of FTD patients from healthy subjects using ASL was reported to be inferior to that with FDG-PET [46], and ALS may not be widely available for use in clinical examination.

\section{SD}

SD is a type of FTLD, and patients show fluent aphasia, anomia, and characteristic impaired comprehension of word meaning. The pathology of SD is heterogeneous, and includes various pathologies [37]. MRI demonstrates predominant anterior and inferior temporal atrophy, usually asymmetrically (particularly on the left side), often accompanied by mild asymmetrical frontal atrophy (Fig. 10). In SD patients, a severely decreased and asymmetric temporal metabolism can be demonstrated on FDG-PET images [10]. These areas can spread to the frontal and parietal cortices, although this deficit is not as marked as in the anterior temporal cortices.

\section{PNFA}

PNFA is characterized by effortful and halting speech production with speech sound errors, and simplified or 'agrammatic' productions, with the main cause related to tauopathy [47]. Regional brain atrophy and decreased cerebral metabolism are seen in the posterior part of the left frontal lobe, including the Broca area and the insula regions [48].

\section{Amyloid imaging and tau imaging in FTLD}

In general, amyloid deposits are uncommon in most FTLD patients. Thus, amyloid PET is an important potential biomarker for differential diagnosis of AD from FTLD [49], and for studying the clinicopathological correlation of amyloid pathology in FTLD [50]. Since recent tau PET tracers have off-target problems involving binding to non-specific tau molecules, tau PET does not indicate pure tau deposition, but rather is used to show non-tau associated with neurodegeneration pathology $[51,52]$ and the correlation of FTLD pathology with function [53]. PNFA may be associated with 


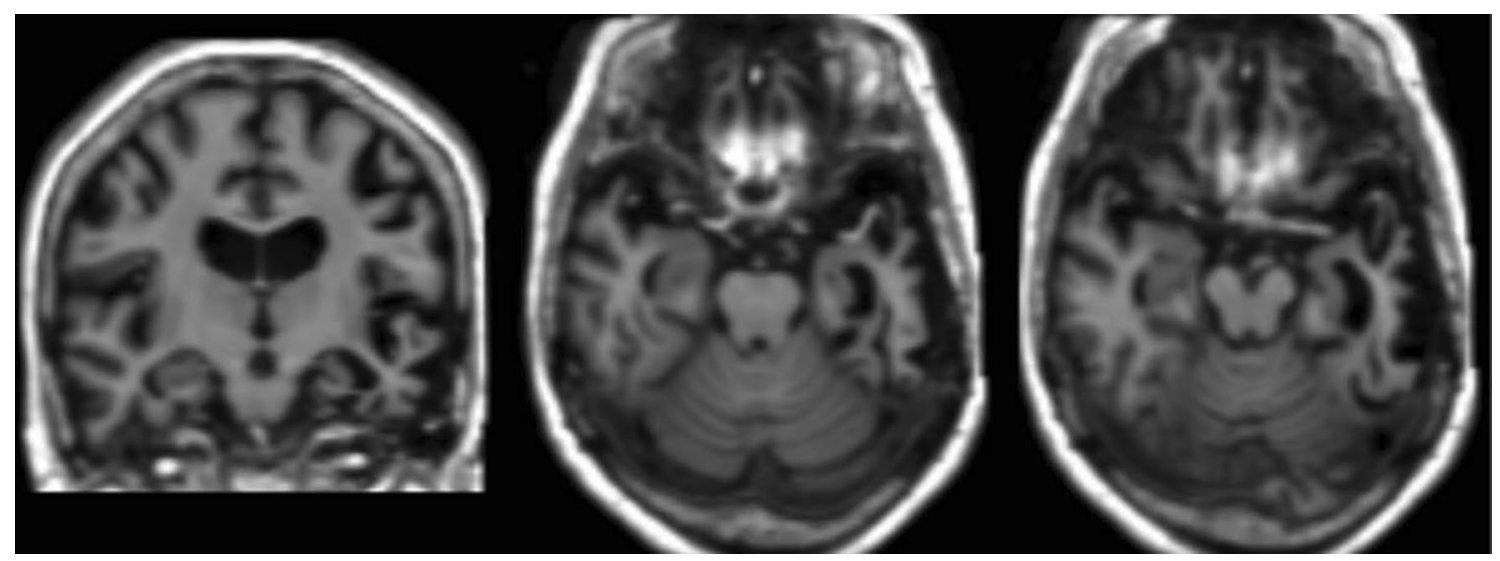

Fig. 10 Representative case of SD. Severe left anterior temporal atrophy is demonstrated on T1-weighted MRI

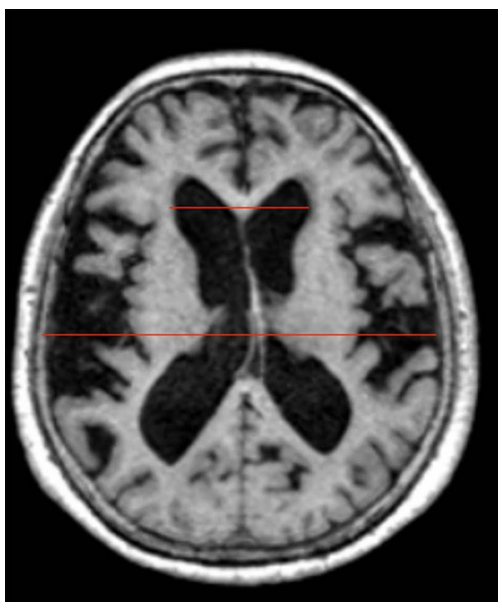

Fig. 11 Evans index (EI). The EI is calculated as the ratio of the maximum width of the frontal horns of the lateral ventricles compared with the maximum width of the internal diameter of the skull at the same level

AD pathology, and amyloid PET can be used as a positive biomarker for $\mathrm{AD}$ [54].

\section{NPH}

NPH is a syndrome characterized by a triad of symptoms involving gait impairment, cognitive decline, and urinary incontinence, and is associated with ventriculomegaly. NPH is classified into idiopathic NPH (iNPH) and secondary NPH. iNPH involves disproportionally enlarged subarachnoid space hydrocephalus (DESH) and non-DESH [55]. Based on iNPH criteria, 'possible iNPH' is diagnosed in patients (a) $>60$ years old; (b) having one or more symptoms of gait disturbance, dementia, and urinary incontinence; (c) with ventricular dilatation (Evans index $>0.3$; Fig. 11) and a narrow cerebrospinal fluid (CSF) space in the superior convexity; (d) with a CSF pressure $<200 \mathrm{mmH}_{2} \mathrm{O}$ with a normal CSF cell count and protein level; (e) having no other diseases that may account for the symptoms; and (f) with no other previous illness that causes ventricular dilatation. 'Probable iNPH' is diagnosed if the spinal tap test is positive with possible iNPH [55].

The characteristic findings of DESH are (a) ventriculomegaly, (b) enlarged Sylvian fissure, and (c) tight sulci in the midline with/without tight sulci at the high convexity (Figs. 12, 13a). Although tight sulci in the high convexity were previously considered important, this finding is not often observed as the lateral sulci are sometimes dilated because of accompanying cortical atrophy. However, tight sulci in the midline, especially the medial parietal sulci, are always seen in DESH. Since iNPH occurs in aged subjects, it can be associated with neurodegenerative dementia such as AD and DLB. In iNPH, the lateral frontal and parietal sulci are dilated because of atrophy, while the midline sulci are always observed. This feature was verified by voxelbased analysis showing that the highest density was in the medial parietal cortex in iNPH $[56,57]$. When estimating the tight sulci, coronal slices have been recommended. However, evaluating the axial images provides a similar or better assessment than that for tight sulci in the midline and a high convexity. Some iNPH cases also show focal dilated sulci. Automatic volume measurement using voxel-based analysis can be used to delineate the characteristic changes in the CSF space in iNPH patients, and is useful for diagnosis [58, 59], although this method is not practical in routine clinical examination.

The callosal angle (CA) [60] (Fig. 14) is a useful index for indirectly demonstrating DESH findings. Most DESH cases show a $\mathrm{CA}<90^{\circ}$ [60]. Moreover, $\mathrm{CA}$ is a good predictor of shunt-response subjects [61, 62]. Recently, other indices for DESH, including the $z$ Evans index [63] and the brain to ventricle ratios [64], have been proposed. The $z$ Evans index was defined as the maximum $z$ axial length of 


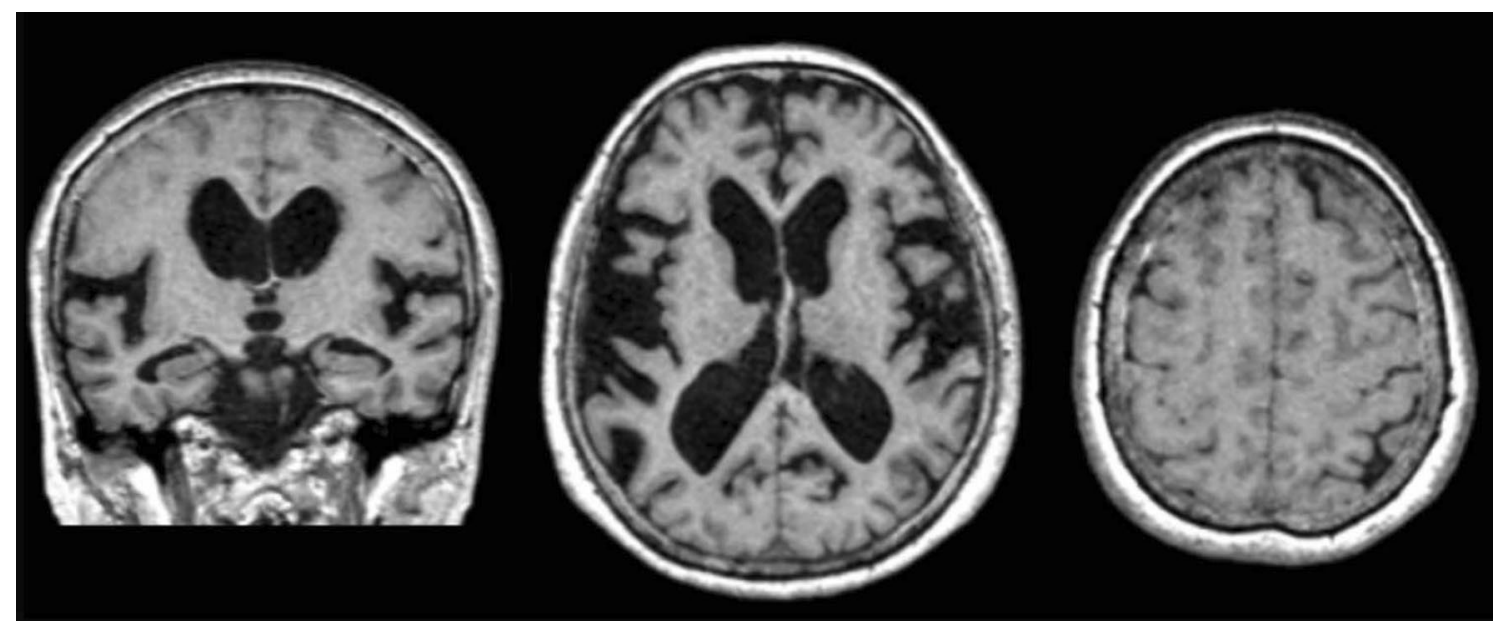

Fig. 12 Representative MRI of disproportionately enlarged subarachnoid space hydrocephalus (DESH). Ventriculomegaly, dilated Sylvian fissures, and tight sulci in the midline and at the high convexity are demonstrated

the frontal horns to the maximum cranial $z$ axial length, and was reported useful for predicting tap-positive subjects with iNPH [63]. The brain to ventricle ratios are calculated as the maximum width of the brain divided by the maximum width of the lateral ventricles at the anterior and posterior commissure levels, respectively, and were reported to be useful for differential diagnosis of iNPH or iNPH with AD from $\mathrm{AD}$, similar to the $\mathrm{CA}$ and the $z$ Evans [64]. Asymptomatic ventriculomegaly with features of iNPH on MRI [65] has the same radiologic features of DESH, including a dilated ventricular system, enlarged Sylvian fissures, and a tight high convexity/midline sulci, but has no clinical symptoms. Thus, it is thought to be a preclinical form of iNPH [65].

Diffusion tensor imaging is also a useful tool for differentiating iNPH from other diseases. Microstructural changes in the corticospinal tract have been reported in iNPH $[66,67]$. For differential diagnosis of iNPH and AD, the sensitivity and specificity of the diffusion tensor imaging index fractional anisotropy in the posterior limb of the internal capsule were $87.5 \%$ and $95.0 \%$, respectively [68]. Despite the utility of these techniques, they are not practical in routine clinical examination.

Brain perfusion SPECT reflects the morphological change of DESH (Fig. 13a), with a relatively increased midline and high convexity perfusion [convexity apparently hyperperfusion (CAPPAH) sign] (Fig. 13b, c) [69], and decreased
peri-Sylvian fissure perfusion. Frontal dominant hypoperfusion is common, although posterior and diffuse hypoperfusion have also been reported [70]. Voxel-based statistical images demonstrate these findings as a 'CAPPAH sign' and a 'check mark sign' (Fig. 13c). Amyloid imaging reveals amyloid deposition in $>50 \%$ of patients with iNPH [71], although this phenomenon does not contribute to diagnosis of iNPH because $>20 \%$ of normal subjects $>80$ years of age show amyloid deposits. Computerized tomographic cisternography is not recommended for clinical examination, because although the sensitivities of CTC findings at the lateral ventricles and brain surface for shunt effectiveness were $81.7 \%$ and $86.7 \%$, respectively, the specificities were $20 \%$ and $0 \%$, respectively [72]. Secondary NPH consists of NPH after subarachnoid hemorrhage, meningitis or other diseases, and NPH caused by congenital or developmental etiologies [55]. The radiological features include a dilated ventricular system, non-dilated Sylvian fissures, and slightly tight sulci at the high convexity and midline.

\section{Conclusion}

Neuroimaging: structural MRI, SPECT, and PET provide characteristic features of DLB, FTLD, and iNPH, and help with the diagnoses of these diseases. 

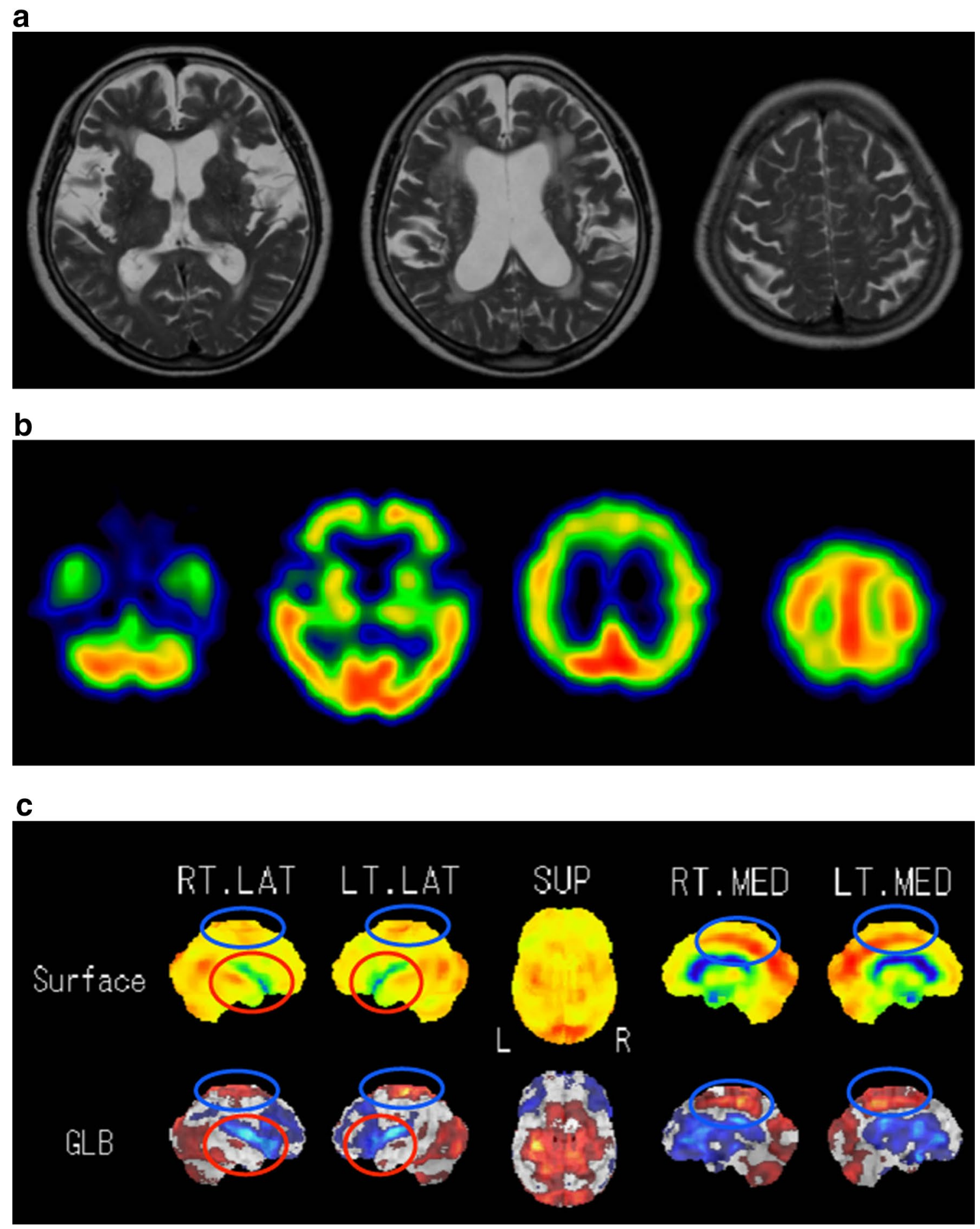

Fig. 13 A representative case of DESH. a T2-weighted axial MRI image showing dilated lateral ventricles, enlarged Sylvian fissures, and tight sulci in the midline, while the lateral frontal and parietal sulci are not tight. b Brain perfusion SPECT showing frontal domi- nant hypoperfusion and midline hyperperfusion. c Voxel-based statistical map. 3D-SSP shows the convexity apparent hyperperfusion (CAPPAH) sign (yellow ellipse) and check mark sign (red ellipse) 

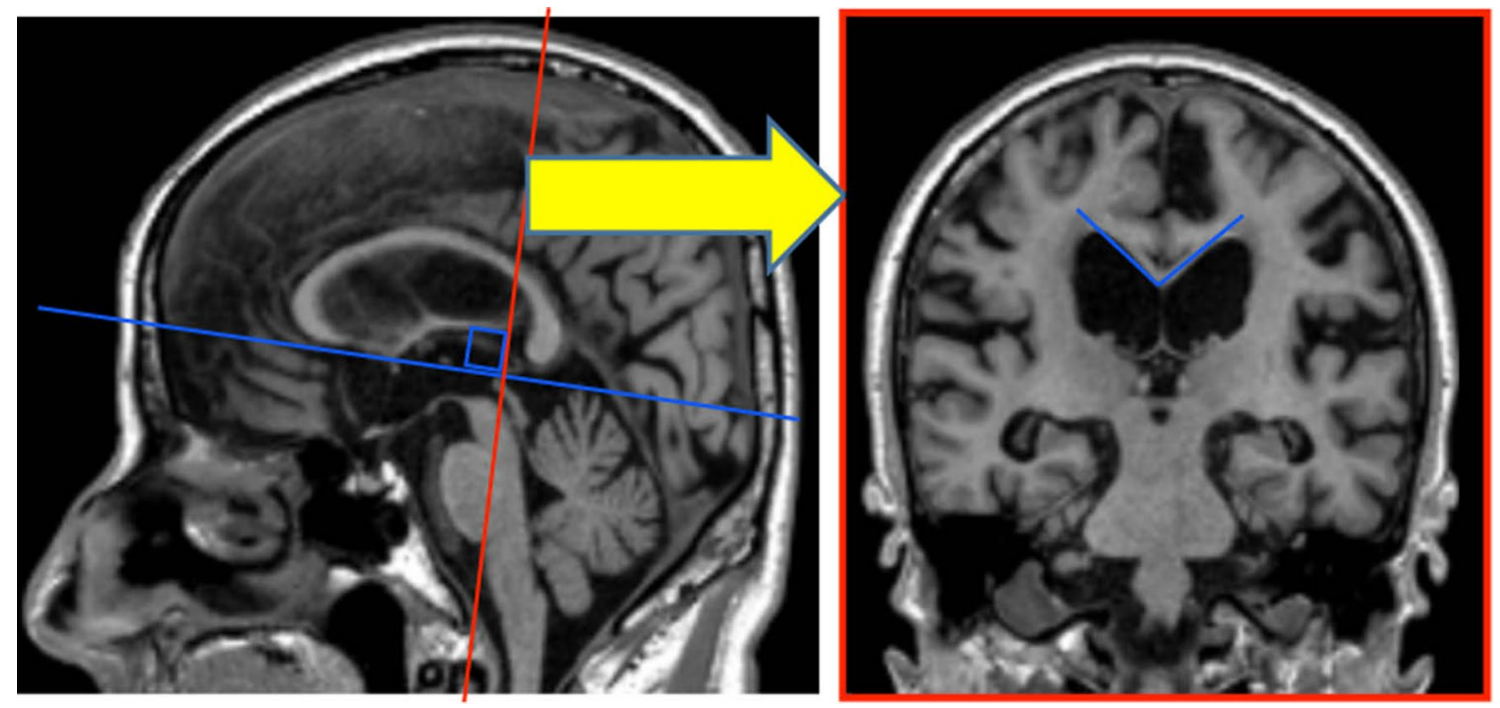

Fig. 14 Callosal angle (CA). The CA is the angle between the lateral ventricles measured on the coronal plane, which is perpendicular to the anteroposterior commissure plane on the posterior commissure of the subject

Acknowledgements This article was aided in part by the Longevity Science Research and Development Project, Ministry of Health, Labour and Welfare, Japan (29-43).

\section{Compliance with ethical standards}

Conflict of interest The authors have no conflicts of interest directly relevant to the content of this article.

Ethical statement This manuscript is a review article and has no studies with human participants or animals performed by any of the authors.

\section{References}

1. Matsuda H, Shigemoto Y, Sato N. Neuroimaging of Alzheimer's disease: focus on amyloid and tau PET. Jpn J Radiol. 2019. https ://doi.org/10.1007/s11604-019-00867-7.

2. McKeith IG, Boeve BF, Dickson DW, Halliday G, Taylor JP, Weintraub D, et al. Diagnosis and management of dementia with Lewy bodies: fourth consensus report of the DLB consortium. Neurology. 2017;89(1):88-100.

3. Hashimoto M, Kitagaki H, Imamura T, Hirono N, Shimomura $\mathrm{T}$, Kazui $\mathrm{H}$, et al. Medial temporal and whole-brain atrophy in dementia with Lewy bodies: a volumetric MRI study. Neurology. 1998;51(2):357-62.

4. Takahashi R, Ishii K, Miyamoto N, Yoshikawa T, Shimada K, Ohkawa $\mathrm{S}$, et al. Measurement of gray and white matter atrophy in dementia with Lewy bodies using diffeomorphic anatomic registration through exponentiated lie algebra: a comparison with conventional voxel-based morphometry. AJNR Am J Neuroradiol. 2010;31(10):1873-8.

5. Nakatsuka T, Imabayashi E, Matsuda H, Sakakibara R, Inaoka T, Terada H. Discrimination of dementia with Lewy bodies from Alzheimer's disease using voxel-based morphometry of white matter by statistical parametric mapping 8 plus diffeomorphic anatomic registration through exponentiated Lie algebra. Neuroradiology. 2013;55(5):559-66.

6. Imamura T, Ishii K, Sasaki M, Kitagaki H, Yamaji S, Hirono $\mathrm{N}$, et al. Regional cerebral glucose metabolism in dementia with Lewy bodies and Alzheimer's disease: a comparative study using positron emission tomography. Neurosci Lett. 1997;235(1-2):49-52.

7. Ishii K, Imamura T, Sasaki M, Yamaji S, Sakamoto S, Kitagaki $\mathrm{H}$, et al. Regional cerebral glucose metabolism in dementia with Lewy bodies and Alzheimer's disease. Neurology. 1998;51(1):125-30.

8. Ishii K, Yamaji S, Kitagaki H, Imamura T, Hirono N, Mori E. Regional cerebral blood flow difference between dementia with Lewy bodies and AD. Neurology. 1999;53(2):413-6.

9. Lobotesis K, Fenwick JD, Phipps A, Ryman A, Swann A, Ballard C, et al. Occipital hypoperfusion on SPECT in dementia with Lewy bodies but not AD. Neurology. 2001;56(5):643-9.

10. Ishii K. PET approaches for diagnosis of dementia. AJNR Am J Neuroradiol. 2014;35(11):2030-8.

11. Ishii K, Hosaka K, Mori T, Mori E. Comparison of FDG-PET and IMP-SPECT in patients with dementia with Lewy bodies. Ann Nucl Med. 2004;18(5):447-51.

12. O'Brien JT, Firbank MJ, Davison C, Barnett N, Bamford C, Donaldson C, et al. ${ }^{18}$ F-FDG PET and perfusion SPECT in the diagnosis of Alzheimer and Lewy body dementias. J Nucl Med. 2014;55(12):1959-65.

13. Chiba Y, Fujishiro H, Iseki E, Kasanuki K, Sato K. The cingulate island sign on FDG-PET vs. IMP-SPECT to assess mild cognitive impairment in Alzheimer's disease vs. dementia with Lewy bodies. J Neuroimaging. 2019. https://doi.org/10.1111/jon.12643.

14. Ishii K, Ito K, Nakanishi A, Kitamura S, Terashima A. Computerassisted system for diagnosing degenerative dementia using cerebral blood flow SPECT and 3D-SSP: a multicenter study. Jpn J Radiol. 2014;32(7):383-90.

15. Lim SM, Katsifis A, Villemagne VL, Best R, Jones G, Saling M, et al. The ${ }^{18} \mathrm{~F}$-FDG PET cingulate island sign and comparison to 
${ }^{123}$ I-beta-CIT SPECT for diagnosis of dementia with Lewy bodies. J Nucl Med. 2009;50(10):1638-45.

16. Imabayashi E, Yokoyama K, Tsukamoto T, Sone D, Sumida K, Kimura Y, et al. The cingulate island sign within early Alzheimer's disease-specific hypoperfusion volumes of interest is useful for differentiating Alzheimer's disease from dementia with Lewy bodies. EJNMMI Res. 2016;6(1):67.

17. Iizuka T, Iizuka R, Kameyama M. Cingulate island sign temporally changes in dementia with Lewy bodies. Sci Rep. 2017;7(1): 14745 .

18. Walker Z, Costa DC, Walker RW, Shaw K, Gacinovic S, Stevens T, et al. Differentiation of dementia with Lewy bodies from Alzheimer's disease using a dopaminergic presynaptic ligand. J Neurol Neurosurg Psychiatry. 2002;73(2):134-40.

19. Takaya S, Sawamoto N, Okada T, Okubo G, Nishida S, Togashi $\mathrm{K}$, et al. Differential diagnosis of parkinsonian syndromes using dopamine transporter and perfusion SPECT. Parkinsonism Relat Disord. 2018;47:15-211.

20. Shimizu S, Hirose D, Namioka N, Kanetaka H, Hirao K, Hatanaka $\mathrm{H}$, et al. Correlation between clinical symptoms and striatal DAT uptake in patients with DLB. Ann Nucl Med. 2017;31(5):390-8.

21. van der Zande JJ, Booij J, Scheltens P, Raijmakers PG, Lemstra AW. [(123)]FP-CIT SPECT scans initially rated as normal became abnormal over time in patients with probable dementia with Lewy bodies. Eur J Nucl Med Mol Imaging. 2016;43(6):1060-6.

22. Watanabe H, Ieda T, Katayama T, Takeda A, Aiba I, Doyu M, et al. Cardiac (123)I-meta-iodobenzylguanidine (MIBG) uptake in dementia with Lewy bodies: comparison with Alzheimer's disease. J Neurol Neurosurg Psychiatry. 2001;70(6):781-3.

23. Oda H, Ishii K, Terashima A, Shimada K, Yamane Y, Kawasaki R, et al. Myocardial scintigraphy may predict the conversion to probable dementia with Lewy bodies. Neurology. 2013;81(20):1741-5.

24. Shimizu S, Hirao K, Kanetaka H, Namioka N, Hatanaka H, Hirose $\mathrm{D}$, et al. Utility of the combination of DAT SPECT and MIBG myocardial scintigraphy in differentiating dementia with Lewy bodies from Alzheimer's disease. Eur J Nucl Med Mol Imaging. 2016;43(1):184-92.

25. Treglia G, Cason E, Cortelli P, Gabellini A, Liguori R, Bagnato A, et al. Iodine-123 metaiodobenzylguanidine scintigraphy and iodine-123 ioflupane single photon emission computed tomography in Lewy body diseases: complementary or alternative techniques? J Neuroimaging. 2014;24(2):149-54.

26. Gomperts SN, Rentz DM, Moran E, Becker JA, Locascio JJ, Klunk WE, et al. Imaging amyloid deposition in Lewy body diseases. Neurology. 2008;71(12):903-10.

27. Donaghy P, Thomas AJ, O'Brien JT. Amyloid PET Imaging in Lewy body disorders. Am J Geriatr Psychiatry. 2015;23(1):23-37.

28. Siderowf A, Pontecorvo MJ, Shill HA, Mintun MA, Arora A, Joshi AD, et al. PET imaging of amyloid with Florbetapir F 18 and PET imaging of dopamine degeneration with 18F-AV-133 (florbenazine) in patients with Alzheimer's disease and Lewy body disorders. BMC Neurol. 2014;14:79.

29. Brooks DJ. Imaging amyloid in Parkinson's disease dementia and dementia with Lewy bodies with positron emission tomography. Mov Disord. 2009;24(Suppl 2):S742-S747747.

30. Kantarci K, Lowe VJ, Boeve BF, Weigand SD, Senjem ML, Przybelski SA, et al. Multimodality imaging characteristics of dementia with Lewy bodies. Neurobiol Aging. 2012;33(9):2091-105.

31. Palermo G, Tommasini L, Aghakhanyan G, Frosini D, Giuntini M, Tognoni G, et al. Clinical correlates of cerebral amyloid deposition in Parkinson's disease dementia: evidence from a PET study. J Alzheimers Dis. 2019;70(2):597-609.

32. Ishii K, Hosokawa C, Hyodo T, Sakaguchi K, Usami K, Shimamoto K, et al. Regional glucose metabolic reduction in dementia with Lewy bodies is independent of amyloid deposition. Ann Nucl Med. 2015;29(1):78-83.
33. Rascovsky K, Hodges JR, Knopman D, Mendez MF, Kramer $\mathrm{JH}$, Neuhaus J, et al. Sensitivity of revised diagnostic criteria for the behavioural variant of frontotemporal dementia. Brain. 2011;134(Pt 9):2456-77.

34. Gorno-Tempini ML, Hillis AE, Weintraub S, Kertesz A, Mendez M, Cappa SF, et al. Classification of primary progressive aphasia and its variants. Neurology. 2011;76(11):1006-144.

35. Lashley T, Rohrer JD, Mead S, Revesz T. Review: an update on clinical, genetic and pathological aspects of frontotemporal lobar degenerations. Neuropathol Appl Neurobiol. 2015;41(7):858-81.

36. Cairns NJ, Bigio EH, Mackenzie IR, Neumann M, Lee VM, Hatanpaa KJ, et al. Neuropathologic diagnostic and nosologic criteria for frontotemporal lobar degeneration: consensus of the Consortium for Frontotemporal Lobar Degeneration. Acta Neuropathol. 2007;114(1):5-22.

37. Seelaar H, Rohrer JD, Pijnenburg YA, Fox NC, van Swieten JC. Clinical, genetic and pathological heterogeneity of frontotemporal dementia: a review. J Neurol Neurosurg Psychiatry. 2011;82(5):476-86

38. McCarthy J, Collins DL, Ducharme S. Morphometric MRI as a diagnostic biomarker of frontotemporal dementia: a systematic review to determine clinical applicability. Neuroimage Clin. 2018;20:685-96.

39. Meyer S, Mueller K, Stuke K, Bisenius S, Diehl-Schmid J, Jessen $\mathrm{F}$, et al. Predicting behavioral variant frontotemporal dementia with pattern classification in multi-center structural MRI data. Neuroimage Clin. 2017;14:656-62.

40. Moller C, Pijnenburg YA, van der Flier WM, Versteeg A, Tijms B, de Munck JC, et al. Alzheimer disease and behavioral variant frontotemporal dementia: automatic classification based on cortical atrophy for single-subject diagnosis. Radiology. 2016;279(3):838-48.

41. Kanda T, Ishii K, Uemura T, Miyamoto N, Yoshikawa T, Kono $\mathrm{AK}$, et al. Comparison of grey matter and metabolic reductions in frontotemporal dementia using FDG-PET and voxelbased morphometric MR studies. Eur J Nucl Med Mol Imaging. 2008;35(12):2227-34.

42. Bruun M, Koikkalainen J, Rhodius-Meester HFM, Baroni M, Gjerum L, van Gils M, et al. Detecting frontotemporal dementia syndromes using MRI biomarkers. Neuroimage Clin. 2019;22:101711.

43. Ishii K, Sakamoto S, Sasaki M, Kitagaki H, Yamaji S, Hashimoto $\mathrm{M}$, et al. Cerebral glucose metabolism in patients with frontotemporal dementia. J Nucl Med. 1998;39(11):1875-8.

44. Buhour MS, Doidy F, Laisney M, Pitel AL, de La Sayette V, Viader F, et al. Pathophysiology of the behavioral variant of frontotemporal lobar degeneration: a study combining MRI and FDGPET. Brain Imaging Behav. 2017;11(1):240-52.

45. Verfaillie SC, Adriaanse SM, Binnewijzend MA, Benedictus MR, Ossenkoppele R, Wattjes MP, et al. Cerebral perfusion and glucose metabolism in Alzheimer's disease and frontotemporal dementia: two sides of the same coin? Eur Radiol. 2015;25(10):3050-9.

46. Anazodo UC, Finger E, Kwan BYM, Pavlosky W, Warrington JC, Gunther M, et al. Using simultaneous PET/MRI to compare the accuracy of diagnosing frontotemporal dementia by arterial spin labelling MRI and FDG-PET. Neuroimage Clin. 2018;17:405-14.

47. Kirshner HS. Frontotemporal dementia and primary progressive aphasia, a review. Neuropsychiatr Dis Treat. 2014;10:1045-55.

48. Routier A, Habert MO, Bertrand A, Kas A, Sundqvist M, Mertz $\mathrm{J}$, et al. Structural, microstructural, and metabolic alterations in primary progressive aphasia variants. Front Neurol. 2018;9:766.

49. Kobylecki C, Langheinrich T, Hinz R, Vardy ER, Brown G, Martino ME, et al. 18F-florbetapir PET in patients with frontotemporal dementia and Alzheimer disease. J Nucl Med. 2015;56(3):386-91.

50. Ryan KA, Hammers D, DeLeon A, Bilen H, Frey K, Burke J, et al. Agreement among neuropsychological and behavioral data 
and $\mathrm{PiB}$ findings in diagnosing frontotemporal dementia. J Clin Neurosci. 2017;44:128-32.

51. Makaretz SJ, Quimby M, Collins J, Makris N, McGinnis S, Schultz A, et al. Flortaucipir tau PET imaging in semantic variant primary progressive aphasia. J Neurol Neurosurg Psychiatry. 2018;89(10):1024-31.

52. Son HJ, Oh JS, Roh JH, Seo SW, Oh M, Lee SJ, et al. Differences in gray and white matter (18)F-THK5351 uptake between behavioral-variant frontotemporal dementia and other dementias. Eur J Nucl Med Mol Imaging. 2019;46(2):357-66.

53. Asghar M, Hinz R, Herholz K, Carter SF. Dual-phase [18F]florbetapir in frontotemporal dementia. Eur J Nucl Med Mol Imaging. 2019;46(2):304-11.

54. Santos-Santos MA, Rabinovici GD, Iaccarino L, Ayakta N, Tammewar G, Lobach I, et al. Rates of amyloid imaging positivity in patients with primary progressive aphasia. JAMA Neurol. 2018;75(3):342-52.

55. Mori E, Ishikawa M, Kato T, Kazui H, Miyake H, Miyajima $\mathrm{M}$, et al. Guidelines for management of idiopathic normal pressure hydrocephalus: second edition. Neurol Med Chir (Tokyo). 2012;52(11):775-809.

56. Ishii K, Kawaguchi T, Shimada K, Ohkawa S, Miyamoto N, Kanda $\mathrm{T}$, et al. Voxel-based analysis of gray matter and CSF space in idiopathic normal pressure hydrocephalus. Dement Geriatr Cogn Disord. 2008;25(4):329-35.

57. Yamashita F, Sasaki M, Takahashi S, Matsuda H, Kudo K, Narumi $\mathrm{S}$, et al. Detection of changes in cerebrospinal fluid space in idiopathic normal pressure hydrocephalus using voxel-based morphometry. Neuroradiology. 2010;52(5):381-6.

58. Ishii K, Soma T, Shimada K, Oda H, Terashima A, Kawasaki R. Automatic volumetry of the cerebrospinal fluid space in idiopathic normal pressure hydrocephalus. Dement Geriatr Cogn Dis Extra. 2013;3(1):489-96.

59. Yamashita F, Sasaki M, Saito M, Mori E, Kawaguchi A, Kudo $\mathrm{K}$, et al. Voxel-based morphometry of disproportionate cerebrospinal fluid space distribution for the differential diagnosis of idiopathic normal pressure hydrocephalus. J Neuroimaging. 2014;24(4):359-65.

60. Ishii K, Kanda T, Harada A, Miyamoto N, Kawaguchi T, Shimada $\mathrm{K}$, et al. Clinical impact of the callosal angle in the diagnosis of idiopathic normal pressure hydrocephalus. Eur Radiol. 2008;18(11):2678-83.

61. Virhammar J, Laurell K, Cesarini KG, Larsson EM. The callosal angle measured on MRI as a predictor of outcome in idiopathic normal-pressure hydrocephalus. J Neurosurg. 2014;120(1):178-84.

62. Grahnke K, Jusue-Torres I, Szujewski C, Joyce C, Schneck M, Prabhu VC, et al. The quest for predicting sustained shunt response in normal-pressure hydrocephalus: an analysis of the callosal angle's utility. World Neurosurg. 2018;115:e717-e722722.
63. Yamada S, Ishikawa M, Yamamoto K. Optimal diagnostic indices for idiopathic normal pressure hydrocephalus based on the 3D quantitative volumetric analysis for the cerebral ventricle and subarachnoid space. AJNR Am J Neuroradiol. 2015;36(12):2262-9.

64. Yamada S, Ishikawa M, Yamamoto K. Comparison of CSF distribution between idiopathic normal pressure hydrocephalus and Alzheimer disease. AJNR Am J Neuroradiol. 2016;37(7):1249-55.

65. Iseki C, Takahashi Y, Wada M, Kawanami T, Adachi M, Kato T. Incidence of idiopathic normal pressure hydrocephalus (iNPH): a 10-year follow-up study of a rural community in Japan. J Neurol Sci. 2014;339(1-2):108-12.

66. Hattori T, Yuasa T, Aoki S, Sato R, Sawaura H, Mori T, et al. Altered microstructure in corticospinal tract in idiopathic normal pressure hydrocephalus: comparison with Alzheimer disease and Parkinson disease with dementia. AJNR Am J Neuroradiol. 2011;32(9):1681-7.

67. Irie R, Tsuruta K, Hori M, Suzuki M, Kamagata K, Nakanishi A, et al. Neurite orientation dispersion and density imaging for evaluation of corticospinal tract in idiopathic normal pressure hydrocephalus. Jpn J Radiol. 2017;35(1):25-30.

68. Kim MJ, Seo SW, Lee KM, Kim ST, Lee JI, Nam DH, et al. Differential diagnosis of idiopathic normal pressure hydrocephalus from other dementias using diffusion tensor imaging. AJNR Am J Neuroradiol. 2011;32(8):1496-503.

69. Ohmichi T, Kondo M, Itsukage M, Koizumi H, Matsushima S, Kuriyama N, et al. Usefulness of the convexity apparent hyperperfusion (CAPPAH) sign in 123I-iodoamphetamine brain perfusion single photon emission computed tomography for the diagnosis of idiopathic normal pressure hydrocephalus. J Neurosurg. 2018;130:398-405.

70. Ishii K, Hashimoto M, Hayashida K, Hashikawa K, Chang CC, Nakagawara J, et al. A multicenter brain perfusion SPECT study evaluating idiopathic normal-pressure hydrocephalus on neurological improvement. Dement Geriatr Cogn Disord. 2011;32(1):1-10.

71. Rinne JO, Frantzen J, Leinonen V, Lonnrot K, Laakso A, Virtanen KA, et al. Prospective flutemetamol positron emission tomography and histopathology in normal pressure hydrocephalus. Neurodegener Dis. 2014;13(4):237-45.

72. Kawaguchi T, Hirata Y, Bundo M, Kondo T, Owaki H, Ito S, et al. Role of computerized tomographic cisternography in idiopathic normal pressure hydrocephalus. Acta Neurochir (Wien). 2011;153(10):2041-8 (discussion 8).

Publisher's Note Springer Nature remains neutral with regard to jurisdictional claims in published maps and institutional affiliations. 